University of Nebraska - Lincoln

DigitalCommons@University of Nebraska - Lincoln

April 2006

\title{
Protein-protein interactions of tandem affinity purification-tagged protein kinases in rice
}

\author{
Jai S. Rohila \\ University of Nebraska - Lincoln \\ Mei Chen \\ University of Nebraska - Lincoln, mchen3@unl.edu \\ Shuo Chen \\ University of Nebraska - Lincoln, schen3@unl.edu \\ Johann Chen \\ University of California, Davis \\ Ronald Cerny \\ rcerny1@unl.edu
}

See next page for additional authors

Follow this and additional works at: https://digitalcommons.unl.edu/chemistrycerny

Part of the Chemistry Commons

Rohila, Jai S.; Chen, Mei; Chen, Shuo; Chen, Johann; Cerny, Ronald; Dardick, Chris; Canlas, Patrick; Xu, Xia; Gribskov, Michael; Kanrar, Siddhartha; Zhu, Jian-Kang; Ronald, P C.; and Fromm, Michael E., "Protein-protein interactions of tandem affinity purification-tagged protein kinases in rice" (2006). Ronald Cerny Publications. 4.

https://digitalcommons.unl.edu/chemistrycerny/4

This Article is brought to you for free and open access by the Published Research - Department of Chemistry at DigitalCommons@University of Nebraska - Lincoln. It has been accepted for inclusion in Ronald Cerny Publications by an authorized administrator of DigitalCommons@University of Nebraska - Lincoln. 


\section{Authors}

Jai S. Rohila, Mei Chen, Shuo Chen, Johann Chen, Ronald Cerny, Chris Dardick, Patrick Canlas, Xia Xu, Michael Gribskov, Siddhartha Kanrar, Jian-Kang Zhu, P C. Ronald, and Michael E. Fromm 
Published in The Plant Journal 46:1 (2006), pp.1-13. doi: 10.1111/j.1365-313X.2006.02671.x Copyright @ 2006 Jai S. Rohila, Mei Chen, Shuo Chen, Johann Chen, Ronald Cerny, Chris Dardick, Patrick Canlas, Xia Xu, Michael Gribskov, Siddhartha Kanrar, Jian-Kang Zhu, Pamela Ronald, and Michael E. Fromm. Published by Blackwell Publishing Ltd.

Submitted August 22, 2005; revised November 7, 2005; accepted November 11, 2005; published online February $28,2006$.

\title{
Protein-protein interactions of tandem affinity purification- tagged protein kinases in rice
}

\author{
Jai S. Rohila ${ }^{1}$, Mei Chen ${ }^{1}$, Shuo Chen ${ }^{1}$, Johann Chen², Ronald Cerny ${ }^{3}$, Chris Dardick², Patrick \\ Canlas $^{2}$, Xia Xu² ${ }^{2}$ Michael Gribskov ${ }^{4}$, Siddhartha Kanrar ${ }^{5}$, Jian-Kang Zhu ${ }^{5}$, Pamela Ronald ${ }^{2}$, and \\ Michael E. Fromm ${ }^{1 *}$
}

${ }^{1}$ Plant Science Initiative, University of Nebraska-Lincoln, Lincoln, NE 68588

${ }^{2}$ Department of Plant Pathology, University of California, Davis, CA 95616

${ }^{3}$ Department of Chemistry, University of Nebraska-Lincoln, Lincoln, NE 68588

${ }^{4}$ Department of Biological Sciences, Purdue University, West Lafayette, IN 47907

${ }^{5}$ Botany and Plant Sciences, University of California, Riverside, CA 92521

${ }^{*}$ Corresponding author. Email: mfromm@unlnotes.unl.edu

\begin{abstract}
Summary: Forty-one rice cDNAs encoding protein kinases were fused to the tandem affinity purification (TAP) tag and expressed in transgenic rice plants. The TAP-tagged kinases and interacting proteins were purified from the T1 progeny of the transgenic rice plants and identified by mass spectrometry. Ninety-five percent of the TAP-tagged kinases were recovered. Fifty-six percent of the TAP-tagged kinases were found to interact with other rice proteins. A number of these interactions were consistent with known protein complexes found in other species, validating the TAP-tag method in rice plants. Phosphorylation sites were identified on four of the kinases that interacted with either 14-3-3 proteins or cyclins.
\end{abstract}

Keywords: Oryza sativa, tandem affinity purification, affinity, protein kinase, proteomics

\section{Introduction}

Cell signaling networks allow plants to dynamically respond to environmental changes with developmental, biochemical and physiological adaptations. Reversible protein phosphorylation is one of the most widely used biochemical mechanisms in cell signaling, and the enzymes responsible, protein kinases and phosphatases, constitute about $5 \%$ of the genes in the Arabidopsis (Tchieu et al., 2003) and rice genomes (http://0-rkd. ucdavis.edu.library.unl.edu/). Protein kinases and phosphatases are critical components of many plant signaling pathways including cold, drought and salt tolerance (Chinnusamy et al., 2004; Teige et al., 2004; Zhang et al., 2004), pathogen recognition for disease resistance (Nurnberger et al., 2004; Rowland et al., 2005), ABA (Fan et al., 2004) and ethylene signaling (Etheridge et al., 2005), light perception (Chen et al., 2004), developmental signaling (Dievart and Clark, 2003), regulation of carbon metabolism (Rolland and Sheen, 2005), and cell cycle regulation (Koroleva et al., 2004). They are often found as constituents of signaling complexes that comprise multiple regulatory proteins and are structured by scaffolding, anchoring or adaptor proteins that organize the regulatory proteins (Burack and Shaw, 2000; Morrison and Davis, 2003; Vondriska et al., 2004). The ability to discover these protein-protein interactions has accelerated with advances in mass spectrometry (MS) and tandem affinity purification (TAP) of tagged protein complexes from yeast (Gavin et al., 2002), insect (Forler et al., 2003) and human cells (Gingras et al., 2005), and plants (Rohila et al., 2004; Rubio et al., 2005).

In order to learn more about protein kinase signaling networks in cereal leaves, we have initiated a project to TAP-tag most of the rice protein kinases that are expressed in leaves for subsequent analysis of interacting proteins in transgenic rice plants. Rice is the most important food crop in the world and is experimentally advantageous because its small 389-Mb genome has been sequenced (Goff et al., 2002; IRGSP, 2005; Yu et al., 2002) and it has efficient plant transformation methods (Sallaud et al., 2003) and excellent genetics, with emerging Tos17 retrotransposon (Miyao et al., 2003; Yazaki et al., 2004) and T-DNA mutant collections (An et al., 2003; Sallaud et al., 2004). These advantages have made rice a model for cereal genomics (Ren- 
sink and Buell, 2004) and proteomics. We report here the analysis of 41 TAP-tagged rice protein kinases purified from rice plants. We found $95 \%$ of the TAP-tagged rice protein kinases can be purified in amounts sufficient for MS analysis, and $56 \%$ of these have been isolated as complexes with one or more interacting proteins. The validity of some of the interacting proteins in the isolated protein complexes is supported by evidence from interactions of homologous proteins in other organisms.

\section{Results}

Rice protein kinase gene expression, $c D N A$ isolation and protein isolation

The protein kinases in the rice genome were annotated using release three of the rice genome annotation (http://www.tigr.org/tdb/e2k1/osa1/)and full length cDNA data (Kikuchi et al., 2003). This set consists of 1429 unique protein kinases (http://0-rkd.ucdavis.edu.library. unl.edu/) with an additional 81 forms due to alternative splicing. As the beginning of our efforts to TAP-tag many of the leaf-expressed rice protein kinases, an initial 41 of the leaf-expressed protein kinases that are representative of the types in the rice genome were chosen for a small-scale analysis. The leaf expression of these genes was confirmed by RT-PCR analysis of mRNA isolated from leaves (data not shown). These protein kinases were cloned as cDNAs into pDONR207, a Gateway ${ }^{\circledR}$ recombination vector (Invitrogen, Carlsbad, CA, USA). In the case of the receptor-like kinases (RLKs), only the intracellular domains of the RLKs were cloned to avoid the difficulties of purifying membrane-bound protein complexes (Gavin et al., 2002). A list of the 41 cloned cDNAs is shown in Table 1. These cDNAs were then recombined into a pCAMBIA-1300 binary plasmid derivative. This binary vector uses the maize ubiquitin promoter and intron (Miki and Shimamoto, 2004) to constitutively express the cDNAs in rice plants and contains a TAP tag for in-frame fusions to the $\mathrm{N}$ terminus of the expressed proteins (Figure 1). Agrobacterium-mediated transformation (Chern et al., 2005; Hiei et al., 1994) was used to transfer each construct into rice calli that were subsequently regenerated into transgenic plants.

Leaves of T0 transgenic rice plants were assayed to determine the expression levels of the TAP-tagged protein kinases using a bead-based assay (Rohila et al., 2004) as an initial screen (data available in Supplementary material) or by Western blot in a few cases. Positive samples were then confirmed by Western blotting as shown in a representative Western blot (Figure 2). T1 progeny from the highest expressing parental lines were planted in the greenhouse and the young shoots from 6to 8-week-old plants were harvested. The TAP-tagged kinases were purified using a previously published TAP procedure for plants (Rohila et al., 2004). The purified samples were resolved by SDS-PAGE, stained with a fluorescent dye and visualized by UV. Two representative purifications are shown in Figure 3 (and additional examples are shown in the Supplementary material). The proteins in the isolated gel bands were digested with trypsin and processed as previously described for MS analysis of protein complexes from plants (Rohila et al., 2004).

\section{Protein contaminants and interacting proteins}

Two mock purifications from non-transgenic rice plants were used as background controls for our standard TAP protocol. These purifications generally recovered a low amount of rice protein that represented a few cellular proteins including ATP synthase (Table 2). These background proteins were also occasionally recovered from purifications of transgenic TAP-tagged protein kinases. Purifications of the 41 TAP-tagged protein kinases identified several additional types of abundant cellular proteins that were also recovered in multiple instances. These include various hsp60- and hsp70-related chaperones, ribosomal proteins, rubisco, glyceraldehyde3-phosphate dehydrogenase, phosphoglycerate kinase and other proteins (Table 2). The HSP70-related proteins in particular were found at approximately stochiometric amounts in about one-third of the purifications, but were undetectable in the remaining purifications. Their presence appears to be due to interactions with specific protein kinases, but it is unclear whether the interaction occurs in vivo or in vitro after cell lysis. The HSP70 proteins were listed in the contaminating proteins to be consistent with the yeast TAP-tag results (Gavin et al., 2002). However, the majority of the background proteins was recovered at fairly low levels and did not significantly interfere with obtaining MS data from other purified proteins. It appears that at least some of these background proteins are occasional contaminants due to unknown variables as their amounts were not reproducible in several replicated samples.

The recurring proteins recovered from the mock purifications or found as recurring abundant cellular proteins in purifications of TAP-tagged protein kinases (Table 2) were not included as interacting proteins even though some may represent genuine kinase interactions. The data remaining after removal of these contaminating proteins represents the proteins interacting with the TAP-tagged protein kinases and are shown in Table 1. Peptide coverage of the proteins in the MS data is partially dependent on the abundance of the isolated protein. Peptide coverage of the identified proteins ranged from a single high-scoring peptide to collections of peptides representing over $60 \%$ of the identified protein. In some cases, a single high-scoring peptide identified proteins predicted to interact with the tagged kinase, based on the known subunits of orthologous protein complex 
Table 1 Tandem affinity purification (TAP)-tagged protein kinases and interacting proteins

1. Wall-associated kinase (WAK) 2-like protein Os07g31130 (7): phosphatidylinositol-specific phospholipase C 0s07g49330 (1; 62 ); IQ calmodulin-binding motif Os04g56740 (1; 58); Expressed protein Os02g42300 (1; 66); Disease resistance Os10g04190 (1; 52); Unknown protein Os03g52690 (1;51), a'Glutamate decarboxylase Os08g36320 (5)

2. Putative WAK 1-like protein Os10g10130 (27): leucine-rich repeat, related to Xa21 Os02g12450 (1; 41); Protein kinase domain, putative Os04g24290 (1; 42); Peroxisome biogenesis protein PEX1 Os08g44240 (1; 40); DEAD/DEAH box RNA helicase Os03g01830 (1;67); Aspartic proteinase Os05g04630 (3); Oxygen evolving enhancer 3 PsbO Os02g36850 (1; 63); Hydroxyproline-rich glycoprotein 1 Os02g40880 (3)

3. Receptor-like protein kinase Os12g44090 (1; 81): a Lecithin-cholesterol acyl transferase Os03g52010 (2); S-locus glycoprotein family Os07g36570 (1; 81); a SOUL heme-binding Os02g33020 (1; 83); SulA cell division inhibitor-like Os02g58790 (1; 66); Salt-stress root rs1 Os01g13210 (2); ${ }^{a}$ CBS domain Os08g22160 (1; 66)

4. Receptor kinase Os03g61060 (43): glutamate decarboxylase Os08g36320 (4); ${ }^{a} 14-3-3$ GF14-b Os04g38870 (22); ${ }^{a} 14-3-3$ GF14-c Os08g33370 (20); a 14-3-3 GF14-d Os11g34450 (14); ${ }^{a}$ 14-3-3 Os03g50290 (16); Plasma-membrane proton-efflux P-type ATPase Os04g56160 (2);

a14-3-3 TaWIN2-like Os08g37490 (17); 14-3-3-like Os01g11110 (4); Cysteine proteinase inhibitor Os01g58890 (2)

5. Receptor-like protein Kinase 4 Os07g35010 (27): ICE-like protease caspase p20 domain Os01g58580 (1; 53); Thioredoxin peroxidase Os02g33450 (1; 44)

6. MAP kinase kinase 1 Os01g32660 (54): ${ }^{a}$ Actin Os01g64630 (4); ${ }^{a}$ Mov34/MPN/PAD-1 family Os04g56650 (1; 65); Glyoxysomal malate dehydrogenase Os03g56280 (2); ${ }^{a}$ Glutathione S-transferase Os08g44400 (1; 58); Cellular retinaldehyde-binding CRAL/TRIO domain Os03g51430 $(1 ; 49)$

7. CDC2+/CDC28-related protein kinase Os05g32600 (34): TFIIH p62 subunit Os08g25060 (12); TFIIH p52 Os04g58350 (10); Cyclin H-1 Os03g52750 (9); CDK-activating kinase assembly factor MAT1 Os11g28350 (2); TFIIH p44 Os04g42990 (1; 86 ); TFIIH p34 subunit Os02g03340 (4); Nucleotide excision repair protein XP-D homolog Os05g05260 (25); Ubiquitin or ubiquitin extension protein Os09g39500 (1; 62)

8. Receptor-like kinase Os01g02580 (17): ${ }^{a} \mathrm{PCl}$ domain Os08g43640 (2); Glycine hydroxymethyltransferase 0s03g52840 (1; 79 ); Unknown Os04g46010 (1; 54); ${ }^{a}$ Annexin Os05g31750 (3); Oxidoreductase, Aldo/keto reductase family Os07g05000 (3);

${ }^{a}$ Mov34/MPN/PAD-1 family Os04g56650 (1; 66); Fructose-bisphosphate aldolase class-I Os06g40640 (1; 46); Unknown Os05g30210 (1; 42); a 14-3-3 TaWIN2-like Os08g37490 (5); ${ }^{a} 14-3-3$ GF14-d Os11g34450 (4); Carbonic anhydrase Os01g45280 (1; 75); ${ }^{\text {CCBS domain }}$ Os08g22160 (1; 45)

9. Receptor S/T kinase Os01g02310 (8): harpin-binding protein 1 Os11g38260 (3)

10. Receptor-like protein kinase 4 Os07g 43570 (34): ${ }^{a}$ Proteasome/cyclosome repeat Os09g15750 (6); $26 \mathrm{~S}$ proteasome regulatory subunit S2 Os02g05340 (5); PCl domain Os09g37000 (3); ${ }^{a} \mathrm{PCl}$ domain Os08g43640 (2); $26 \mathrm{~S}$ proteasome subunit Os04g36700 (3); ${ }^{\mathrm{a}} \mathrm{Actin}$ Os01g64630 (2); a Glutathione S-transferase Os08g44400 (2); $26 \mathrm{~S}$ proteasome non-ATPase regulatory subunit Nin1/mts3 Os07g25420 (1; 67); Glutathione S-transferase Os10g38780 (1; 78)

11. Cyclin-dependent kinase B1-1 Os01g67160 (46): actin Os03g50890 (4); 26s proteasome non-ATPase regulatory subunit 6

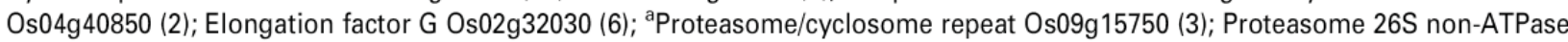
subunit 2 Os04g51910 (2)

12. Ste20-related protein kinase Os10g37480 (17): mapkk or mapkkk similar to WNK1 but has catalytic K Os07g38530 $(1 ; 65) ;{ }^{a} A n n e x i n$ Os05g31750 $(1 ; 51)$

13. rCDC2-1 Os03g02680 (18): cyclin Os03g27420 or Os03g10650 (1; 44); Cyclin D1 Os07g37010 (8); Cyclin D1 Os09g21450 (1; 71 ); Cyclin D1 Os08g32540 (1; 75); Unknown Os09g12000 (1; 53); Cyclin-dependent kinase inhibitor Os02g52480 (4); Cyclin-dependent kinase inhibitor Os10g33310 (1; 105); Unknown Os07g12780 (2); Heat shock protein cognate 70 Os03g60620 (13)

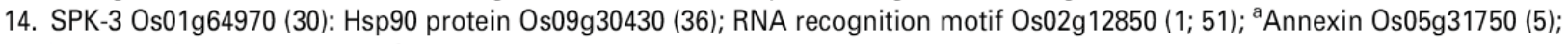
Macronuclear development Os03g52490 $(1 ; 46)$

15. Protein kinase Os01g10450 (39): ${ }^{a} 14-3-3$ Os03g50290 (2); ${ }^{a}$ Glutamate decarboxylase Os08g36320 (2); ${ }^{a} 14-3-3$ GF14-b Os04g38870 (27); a 14-3-3 GF14-d Os11g34450 (17); ${ }^{a}$ 14-3-3 TaWIN2-like Os08g37490 (46); ${ }^{a} 14-3-3$ GF14-c Os08g33370 (33); CBS domain Os09g02710 (1; 74); Thioredoxin Os08g29110 (2); Histone H2A Os10g28230 $(1 ; 60)$

16. Receptor S/T protein kinase Os01g01410 (6): luminal-binding Os03g50250 (2); Acetyl-CoA synthetase Os05g37500 (1; 44); 'Lecithincholesterol acyl transferase Os03g52010 $(1 ; 61)$

17. Casein kinase Os01g38950 (26): SLL2-S9 Os01g49250 (1; 120); ${ }^{a}$ Annexin Os05g31750 (1; 45); MAPK Os08g06060 (3)

18. S-receptor kinase Os01g12410 (4): multidrug resistance-associated protein MRP1 Os01g25380 (1; 43)

19. Protein kinase Os08g12750 (0): armadillo/beta-catenin-like repeat Os10g05730 (1; 80)

20. Casein kinase II (CK2) alpha sub-unit Os07g02350 (27): CK2 regulatory beta subunit Os07g31280 (6); CK2 alpha subunit Os03g10940 (14); CK2 beta subunit Os10g41520 (5); Polyubiquitin 2 Os09g25320(2); RNA-binding region RNP-1 and splicing factor Os08g32100 (1; 86)

21. Protein kinase APK1B Os01g41870 (5): NB-ARC domain Os01g25630 (1; 45); Unknown Os06g51000 (1; 43 ); Unknown Os08g30650 (1; 44 ); aSOUL heme-binding Os02g33020 $(1 ; 45)$

22. Protein kinase Os03g05470 (4): unknown Os10g32120 (1; 43)

23. Wall associated protein kinase Os11g46950 (8): subtilisin-like protease Os01g64860 (1; 51); Tubulin beta-4 chain Os06g46000 (4); Alpha-tubulin Os03g51600 (3); ${ }^{a}$ Actin Os01g64630 (1; 103); Protein kinase domain Os05g40180 (2); Armadillo/beta-catenin-like repeat Os08g43500 (1; 57); Succinate dehydrogenase, flavoprotein subunit Os07g04240 (1; 101); DEAD/DEAH box helicase 0s01g43120 (1; 74) 
Table 1 Continued

Associated proteins not detected for the following protein kinases:

24. Putative wall-associated S/T Kinase Os09g29520 (22); 25. Putative S/T Kinase Os07g48100 (0); 26 . CBL-interacting protein kinase 2 Os01g10890 (19); 27. LRR receptor-like protein kinase Os01g02040 (44); 28. Wall-associated kinase 2-like protein Os07g31190 (2); 29. Receptor S/T Kinase Os01g02290 (2); 30. S/T Prot kinase Mak male germ cell associated kinase Os06g02550 (4); 31 . Putative S/T protein kinase Os07g32400 (8); 32. Putative S/T protein kinase Os01g60910 (5); 33. MAP kinase Os03g17700 (87); 34 . Protein kinase A FC1 Os01g62080 (3); 35. Receptor protein kinase Os07g38070 (3); 36. Receptor-like kinase Os01g02390 (10); 37 . Shaggy-related protein kinase gamma Os01g14860 (22); 38. SAPK2 GI: 22296424 (36); 39. Protein kinase Os03g50330 (9); 40. Calcium dependent Protein kinase Os07g22710 (29); 41. Protein kinase Os04g52860 (25)

The number of peptides identified by MS analysis for each protein is shown in parentheses. In the cases where only a single peptide was identified, the second number in the parentheses is the MASCOT score for that peptide.

andicates interacting proteins found in more than one protein complex but annotated as interacting.

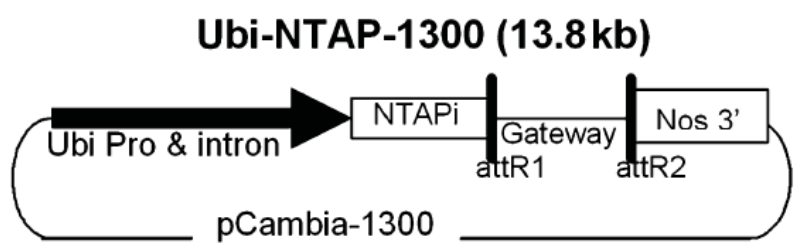

Figure 1. Structure of the Ubi-NTAP-1300 vector.

The maize ubiquitin promoter and intron (Ubi Pro and intron) and nopaline synthase 3' polyadenylation region (Nos 3') are used to express the protein kinase cDNAs after they are recombined into the Gateway ${ }^{\circledR}$ portion of the plasmid between the attR1 and attR2 recombination sites. The NTAPi region (NTAPi) is used to create an upstream fusion to the $\mathrm{N}$ terminus of the introduced protein kinases.

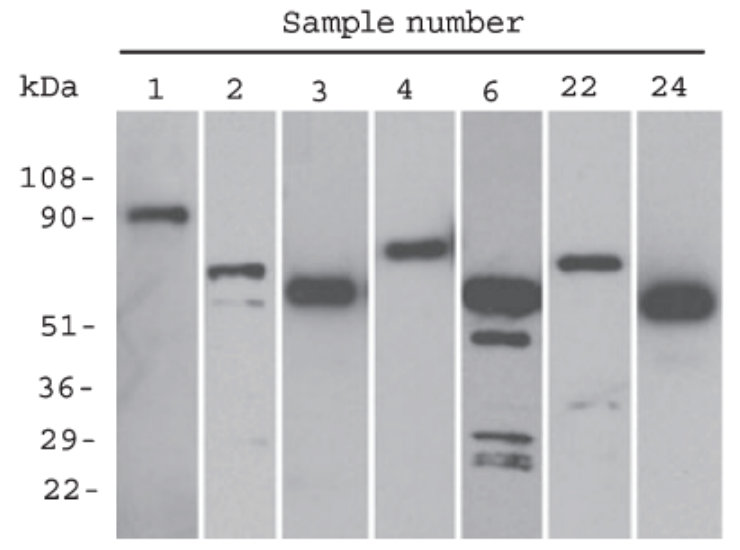

Figure 2. Western blot of TAP-tagged rice protein kinases in transgenic rice plants.

Extracts were prepared from leaves of T0 transgenic plants and the TAP-tagged protein bound to calmodulin-agarose beads. The proteins bound to the beads were analyzed by Western blot, using an antibody: peroxidase probe. The sample number refers to the numbers in Table 1.

es. This indicates that this level of coverage can be meaningful. Some of the specific interactions will be discussed in the Discussion section.

Table 3 shows the summary of the percentages of successfully purified TAP-tagged protein kinases and of the associated protein complexes. Thirty-nine of the 41 TAP-tagged protein kinases were recovered and 23

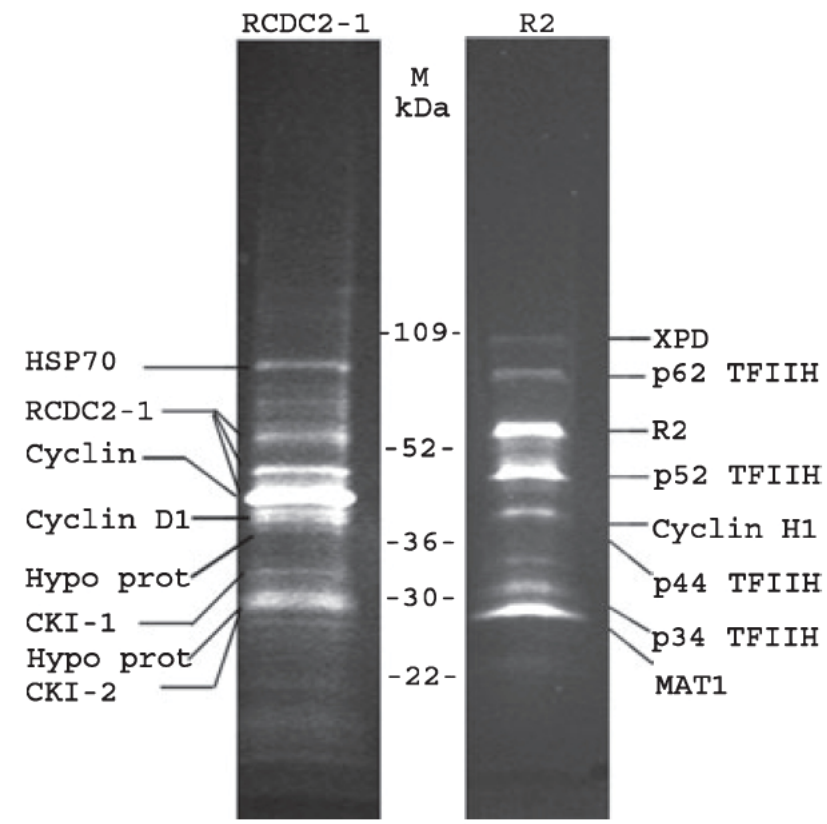

Figure 3. Fluorescently stained TAP-purified proteins after SDSPAGE.

TAP-tagged protein complexes were purified from transgenic rice plants, separated by SDS-PAGE, and visualized with Sypro Ruby fluorescent stain. RCDC2-1 (Table 1, no. 13) is a rice cyclin-dependent kinase (CDK) that controls cell division; R2 (Table 1, no. 7) is a rice CDK-activating kinase (CAK). The names of the proteins in the bands that were identified by mass spectrometry (MS) analysis of the digested peptides are shown. M, markers; hypo. prot.; hypothetical protein; $\mathrm{CKI}$, cyclin-dependent kinase inhibitor; TFIIH, general transcription factor IIH; XPD, nucleotide excision repair protein XP-D homolog.

kinases showed interactions after subtracting the common contaminating proteins (Table 2).

Phosphorylation sites of kinases interacting with 14-3-3, rCDC2-1 or R2

Five kinase complexes contained interacting proteins that are known or anticipated to associate in a phosphorylation-dependent manner or have potential phosphorylation sites. Three complexes contained 14-3-3 proteins (Table 1, nos 4, 8, 15). As more fully described in the dis- 
Table 2 Recurring proteins subtracted from the interacting protein list

\section{Proteins found in mock purifications (two mock preps)}

Hypothetical protein Os06g12930; Hypothetical protein Os10g21540; ATP synthase F1 subunits; DREPP2 protein Os02g18410

Types of recurring common cellular proteins (46 tandem affinity purification (TAP)-tagged preps)

Hsp60 and hsp70-related chaperones; Ribosomal proteins; Rubisco; Translational elongation factor Tu Os02g38210; Translation elongation factor EF-1 Os03g08010; Oxidoreductase Os04g58200; Endopeptidase Clp Os05g51450; Phosphoglycerate kinase Os05g41640; Glyceraldehyde-3-phosphate dehydrogenase; Expressed protein Os05g37400; Hypothetical protein Os10g21540; Putative S-receptor kinase Os10g05160; Retrotransposon protein Os03g12600; Exostosin family Os03g05110; Potassium channel protein Os05g35410; RuBisCO activase Os11g47970;

Table 3 Purification and interaction percentages of tandem affinity purification (TAP)-tagged protein kinases

\begin{tabular}{llr}
\hline & Number & $\%$ \\
\hline Expressed TAP-tagged protein kinases & 41 & 100 \\
Recovered TAP-tagged protein kinases & 39 & 95 \\
Identified complexes & 23 & 56 \\
\hline
\end{tabular}

cussion section, many 14-3-3 proteins are known to bind phosphorylated proteins. The other two complexes contained either the cyclin-dependent kinase (CDK) rCDC21 (Table 1, no. 13) or the CDK-activating kinase (CAK) R2 (Table 1, no. 7). These are expected to be regulated, in part, by protein phosphorylation or dephosphorylation at their ATP binding sites and T-loop regions. The MS data were examined for phosphorylated peptides derived from the complexes containing 14-3-3 proteins as potential 14-3-3 binding sites. The MS data were also examined for the phosphorylation status of the ATP binding site and the T-loop regulatory regions of the R2 (Table 1, no. 7) and rCDC2-1 (Table 1, no. 13) proteins. Phosphorylated peptides were found in four of the five complexes (Table 4) and it is possible that ion suppression of phosphorylated peptides might have prevented their detection in the cases where they were not found, contributing to the weak signals found in two of the peptides. Three peptides contained unique serine or threonine amino acids in the peptide that allowed for assignment of the phosphorylation site. The remaining peptides contained multiple potential phosphorylation sites but only one phosphorylated amino acid was identified in the MS data and is shown for each peptide in Table 4.

Phosphorylated peptides from the three kinase complexes that contained 14-3-3 proteins were examined for potential 14-3-3 binding sites (Table 4). The consensus sequences for 14-3-3 protein binding have been determined to be either RSX[pS/pT]XP or $\mathrm{RXZ}[p S / p T] \mathrm{XP}$, where $\mathrm{pS}$ or $\mathrm{pT}$ represents a phosphorylated serine or threonine amino acid and $Z$ represents an aliphatic amino acid, most often tyrosine or phenylalanine in this consensus sequence (Yaffe et al., 1997). Six of the seven phosphopeptides identified that might bind to 14-3-3 proteins contain two or three of the four consensus amino acids. The amino acids matching the consensus are underlined in Table 4. These homologies to the 14-3-3 protein consensus binding sequence strengthen the possibility that the 14-3-3 proteins are binding one or more of these phosphorylated peptide sequences.

The rCDC2-1 and R2 kinases are known to be regulated by phosphorylation at two positions. Phosphorylation at the threonine and tyrosine amino acids at the ATP binding sites inactivate the kinases while dephosphorylation at these positions is necessary for active enzymes (Morgan, 1995). Our MS data identified only dephosphorylated forms of the peptides spanning these regions (Table 4), suggesting that at least some of the proteins could be active, although ion suppression of any corresponding phosphorylated peptides precludes determining the percentage that is active. Additionally, activation of these enzymes requires that the Thr in the T-loop region be phosphorylated. We did not recover the T-loop region for the rCDC2-1 protein, so its phosphorylation status could not be determined. Our MS data showed the peptide spanning the T-loop region and an adjacent peptide to be phosphorylated in R2 (Table 4). These peptides (IFGpSPER and NFpTHQVFAR) contain unique serine or threonine amino acids indicating these are the sites of phosphorylation, in agreement with the MS data. Phosphorylation of either of these amino acids in the analogous position in the Drosophila CDK7 protein is required for binding cyclins (Larochelle et al., 2001) An additional peptide in the carboxylterminal region of $\mathrm{R} 2$ was also found to be phosphorylated but has an unknown function (Table 4).

\section{Discussion}

Our results indicate the TAP method in transgenic rice plants recovers interacting protein complexes at frequencies similar to those reported in yeast. The success rate of purifying protein complexes from 874 expressed TAP-tagged yeast proteins was $52 \%$ for complexes with one or more interacting proteins (Gavin et al., 2002). This is very similar to our frequency of $56 \%$, although we had a higher frequency of recovering the tagged protein (95\% in plants versus $67 \%$ in yeast) and a lower frequency of recovering protein complexes from successful purifications (58\% in plants and $78 \%$ in yeast). The higher frequency of recovering complexes from purifications judged successful in yeast could be due to elimination of the competition by the native protein through the homologous replacement of the endogenous gene (Gavin et al., 2002), while in our experiments both the 


\begin{tabular}{lll}
\hline TAP-tagged protein kinase & & \\
\hline (Peptide role) & Peptide & Phosphorylation $^{\text {a }}$ \\
\hline Receptor-like kinase & & \\
Os03g61060; Table 1, no. 4 & & \\
(Binds 14-3-3 ?) & K/RQFpSTTSQGK & Yes \\
(Binds 14-3-3 ?) & R/YOpSCPPEVYR & Yes \\
(Binds 14-3-3 ?) & R/QApSSYSVPSEISR & Yes \\
Receptor-like kinase & & \\
Os01g02580 Table 2, no. 8 & & R/DOSFVPLpSALR \\
(Binds 14-3-3 ?) & & \\
Protein Kinase & & Weak \\
Os01g10450 Table 1, no. 15 & R/SKpSPLPNVAPSDVFR \\
(Binds 14-3-3 ?) & K/ADFDIPSpSPPVNSVNS & Yes \\
(Binds 14-3-3 ?) & R/SLVKSPpSSAMLLSYLNK & Weak \\
(Binds 14-3-3 ?) & & \\
R2 CAK & & No \\
Os05g32600 Table 1, no. 7 & EVLGEGTYGVVFK & Yes \\
(ATP binding) & NFpTHOVFAR & Yes \\
(T-loop) & IFGpSPER & Yes \\
(T-loop) & IPDLNLQDGPVVLpSPPR & \\
(Unknown function) & & No \\
rCDC2-1 & & \\
Os03g02680 Table 1, no. 13 & IGEGTYGVVYR & \\
(ATP binding) & Not detected & \\
(T-loop) & & \\
\hline
\end{tabular}

The possible biological role of the peptide site that is regulated by phosphorylation is in parentheses and the phosphorylated amino acid identified is shown in bold as a pS or pT when Ser or Thr are phosphorylated, respectively. Amino acids that match the consensus binding site for 14-3-3 proteins are underlined (consensus is RSX[pS/pT]XP or RXZ[pS/pT]XP, where Z represents aromatic or aliphatic amino acids). The amino acid upstream of the trypsin cleavage site is shown for the putative 14-3-3 protein binding sites, where the cleavage site is shown as forward slash ( $/$.

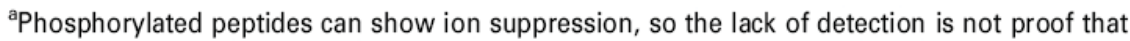
phosphorylated species are absent.

endogenous gene and the expressed TAP-tagged gene were present. The use of RNAi to reduce the expression of the endogenous protein, which can compete with the tagged protein in the formation of the protein complex, has been shown to be beneficial in insect cells (Forler et al., 2003). In plants, TAP-tagged methods could be optimized by complementing null mutants, made either through genetic or reverse-genetic methods. However, given that plant kinases are often members of large gene families (Shiu et al., 2004), obvious phenotypes are not typically observed for many insertion mutations, presumably due to proteins with functional redundancy that may also compete in the formation of a complex. An RNAi-based approach is confounded by the need to silence the endogenous gene(s) without silencing the expressed cDNA. Consequently, the overexpression approach used here is a reasonable approach for obtaining a protein complex with the tagged protein when the above options are not readily available.

When an endogenous gene is present, the overexpression approach potentially allows the tagged protein to better compete with the endogenous protein, as was demonstrated with overexpressed FLAG-tagged proteins
Table 4 Phosphorylation status of kinases binding to $14-3-3$ proteins or cyclins in yeast (Ho et al., 2002). Constitutive expression of the 41 TAP-tagged cDNAs in leaves is consistent with the leaf expression we observed from their corresponding endogenous genes. These cDNAs are constitutively expressed in the transgenic rice plants using the highly transcribed maize ubiquitin promoter (Christensen and Quail, 1996). Despite the use of the highly active ubiquitin promoter, expression levels of the protein kinases are low, estimated at $10^{-5}$ to $10^{-6}$ of the total protein, based on the assumption of a $20 \%$ recovery of the purified TAP-tagged protein (Rohila et al., 2004). These low levels could be due to posttranslational regulation of the amount of protein.

At least two limitations may affect the efficacy of using overexpressed TAP-tagged proteins. First, some fusion proteins may not compete well with the endogenous protein, presumably due to steric hindrance from the TAP-tag domain. Such cases might benefit from testing both the $\mathrm{N}$ - and $\mathrm{C}$-terminal fusions as one may occasionally perform better than the other (Rubio et al., 2005), although the scale of the planned experiments precludes testing both versions. In addition, functionality of TAP-tagged proteins is often difficult to assess: particularly in the absence of obvious mutant 
phenotypes. Genetic complementation tests of seven Arabidopsis mutants with the corresponding TAPtagged genes found three could partially complement and only two could fully complement the mutations (Rubio et al., 2005). However, as shown by interaction studies of protein domains and peptides in yeast, it is likely that some protein interactions can still occur in proteins that lack full functionality as measured by complementation assays. We have used this domain approach when TAP-tagging only the intracellular domain of the transmembrane RLKs with apparent success. This choice was made to reduce the reported difficulties of purifying membrane proteins from yeast (Gavin et al., 2002). However, it is possible some interactions that are dependent on the extracellular domain are missed when using this strategy.

The TAP method has been shown to have a $70 \%$ reproducibility for recovering valid interactions (Gavin et al., 2002). Our analysis of five replicated purifications agree with this estimate and find a higher reproducibility for the proteins that are abundant in a complex and a lower reproducibility of proteins that are of lower abundance in the complex. An advantage of the TAP procedure is the high degree of purification and the repeated use of the same purification protocol. This facilitates creating a database of co-purifying contaminating proteins that can be subtracted from the data (Gavin et al., 2002). Abundant cellular proteins judged to be occasional non-specific contaminants include ribosomal proteins, hsp60- and hsp70-related chaperone proteins, and abundant cellular enzymes such as Rubisco, ATP synthase and a few other common proteins (Table 2). Fortunately, the TAP purification method reduces these non-specific contaminants to levels that are generally less than the amounts of the specifically purified proteins. This facilitates obtaining the MS data of the proteins of interest despite the occasional presence of non-specific contaminants.

The HSP70-related proteins appear in one-third of the protein purifications. These HSP70-related proteins are present in almost stochiometric amounts in these purifications while being undetectable in the remaining purifications. This suggests that these interactions are protein specific, but it is not clear whether these interactions occur in vivo or in vitro. In our previous analysis of the TAP-tagged chimeric glucocorticoid-receptor transcription factor, we found HSP70 in the purified protein complex. We assigned it a role in the protein complex on the basis of independent reports of its involvement with the glucocorticoid receptor (Pratt et al., 2001) and its increased abundance in a purified protein complex after in vivo cross-linking with formaldehyde (Rohila et al., 2004). However, we have assigned the HSP70-related proteins as likely contaminants, unless supported by independent data, as additional data supporting an in vivo interaction are not available in the current study.
As described in the next section, we were able to validate a number of our protein complexes as containing subunits that are predicted from the subunits of orthologous protein complexes, or to have other independent support, or to be members of proteins known to bind other proteins. Proteins in the latter case include the 14-33 proteins (three of 41 purifications) and annexins (four of 41 purifications). Annexins show a $\mathrm{Ca}^{2+}$-dependent association with membrane phospholipids and are involved in the organization of membrane-associated protein networks and cell signaling (Gerke et al., 2005). In Arabidopsis, mutations in either the AnnAt1 or AnnAt4 annexin genes were found to increase sensitivity to osmotic stress and ABA in a $\mathrm{Ca}^{2+}$-dependent manner (Lee et al., 2004). The presumed occurrence of frequent valid interactions prevents the use of frequency alone for distinguishing the contaminants from the common interacting proteins in the cell, particularly when focusing on a single family of proteins such as the protein kinases. The accumulating information in databases from multiple species on known interactions for homologous proteins facilitates these distinctions.

\section{Cyclin-dependent kinases rCDC2-1 and R2}

The cyclin-dependent kinases are evolutionarily conserved regulators of cell division (Inze, 2005). Their activation requires interaction with a cyclin and phosphorylation by a CAK, and their activity is inhibited by binding of cyclin-dependent kinase inhibitors (CKls). The rice rCDC2-1 CDK is homologous to the yeast and human CDK (CDC2) proteins with a well-conserved PSTAIRE domain. Metazoan CDC2 proteins interact with D cyclins and CKIs, and sometimes with Hsp70 (Diehl et al., 2003). We found the rice rCDC2-1 forms a heterocomplex containing a population of proteins consisting of two cyclins, three cyclin D1 isoforms, two CKI isoforms, and Hsp70 (Table 1, no. 13; Figure 3). The in vivo interactions with subunits that are homologous to those of known protein complexes support the suggestion that $r C D C 2-1$ protein is orthologous to the yeast and human CDC2 proteins and involved in the $\mathrm{G} 1 /$ $\mathrm{S}$ and G2/M transitions in cell division in rice (Inze, 2005). The presence of the CKI proteins in the rCDC2-1 protein indicates some or all of these complexes might be inhibited by the CKI subunits, possibly to facilitate endoreplication of the leaf cells through an alternative endoreplication-specific CDK complex (Verkest et al., 2005). The phosphorylation status of the protein was described in the Results section and was found to be consistent with a potentially active state of the rCDC2-1 protein kinase.

The rCDC2-1 protein is expected to be regulated by phosphorylation by a CAK, and the rice R2 CAK is a possible candidate. $\mathrm{R} 2$ is homologous to the metazoan CDK7 CAK that forms a heterotrimeric complex composed of the CDK7 kinase subunit, a regulatory cyclin $\mathrm{H}$ subunit and an assembly factor MAT1. Additionally, the human CDK7 
protein has been shown to phosphorylate the C-terminal domain (CTD) of the large subunit of RNA polymerase II as part of a protein complex that forms the general transcription factor IIH (TFIIH; Lee et al., 2005). The two helicase subunits Xeroderma pigmentosum B (XPB), Xeroderma pigmentosum $D(X P D)$ help bind the three subunit CAK complex to the four subunit core of TFIIH (P34, p44, p52, p62) to form the complete nine subunit complex TFI$\mathrm{IH}$ (Zurita and Merino, 2003). R2 in rice has been shown to be nuclear localized and regulated by the cell cycle (Fabian-Marwedel et al., 2002), to phosphorylate CDKs and the CTD of the RNA polymerase II large subunit in vitro, and to interact with cyclin $\mathrm{H}$ in yeast two-hybrid assays (Yamaguchi et al., 1998, 2000). Our finding that R2 interacts in vivo with the rice orthologs of cyclin H, MAT1, and five of the six expected subunits of transcription factor IIH verifies the role of R2 as a plant ortholog of metazoan CDK7 (Table 1, no. 7; Figure 3). This interpretation is strengthened by the phosphorylation found at conserved Ser and Thr residues in the T-loop region. These phosphorylation results, together with the cyclin and MAT1 subunits present in the protein complex, suggest that the R2 complex could be active in rice shoots. The identification of rCDC2-1 and R2 as participating in complexes that are orthologous to known protein complexes indicates that R2 is likely to phosphorylate rCDC2-1 to regulate cell division and also fulfill its other roles in transcription and DNA repair. The one subunit not observed was the XPB helicase for reasons that are not clear. The ubiquitin protein identified in the complex may be due to the likely E3 ubiquitin ligase activity of the Ssl1 TFIIH subunit (Takagi et al., 2005).

\section{4-3-3 proteins}

The 14-3-3 proteins are highly conserved acidic proteins that play a diverse role in regulating metabolic enzymes, regulatory proteins and protein interactions (Ferl, 2004). Arabidopsis has 13 genes in the epsilon and nonepsilon subgroups, while rice contains eight members in the epsilon family. 14-3-3 proteins bind to target proteins as homodimers or heterodimers, typically to proteins phosphorylated at serine or threonine flanked by conserved amino acids. 14-3-3 proteins affect the regulation of phosphorylated proteins when bound and have been shown to be necessary for the regulation of several proteins including the inactivation of nitrate reductase (Bachmann et al., 1996) and the activation of tryptophan hydroxylase (Ichimura et al., 1987). It is predicted that 14-3-3 proteins interact with a large number of proteins involved in protein regulation and signaling in rice (Ferl, 2004). In several instances, we found that $14-3-3$ proteins appear to bind the TAP-tagged protein kinase directly, as both the 14-3-3 proteins and the protein kinases were the most abundant protein components of the purified protein complex, as measured by protein abundance on the SDS-polyacrylamide gels. In the three protein complexes containing 14-3-3 proteins, phosphorylated peptides could be detected in MS analysis of the peptides of the target protein kinases and most of these peptides contained sequences similar to the consensus 14-3-3 binding sites (Table 4). It seems reasonable to speculate that the combined phosphorylation and bound 14-3-3 proteins affect the activity of these kinases, whose roles are currently unknown.

Two, five or six isoforms of the 14-3-3 proteins were uniquely identified in the three different complexes where 14-3-3 proteins were recovered (Table 1, nos 4, $8,15)$. In at least those cases, multiple 14-3-3 isoforms can bind to the same protein target although these isoforms might have different cellular and subcellular locations in the leaf tissues (Paul et al., 2005), and the family members identified constitute up to six of the eight 14-3-3 proteins encoded in the rice genome. The presence of 14-3-3 proteins as heterodimers that can have two distinct binding sites might allow a single subunit to provide the binding specificity.

\section{CK2}

Protein kinase CK2, also known as casein kinase II, occurs as a stable tetrameric complex of two alpha kinase subunits and two beta regulatory subunits. CK2 is involved in a variety of cellular processes including cell cycle and stress responses (Bibby and Litchfield, 2005). In plants, CK2 appears to be involved in a number or processes including circadian clock regulation in Arabidopsis (Daniel et al., 2004), photoperiod sensitivity in rice (Takahashi et al., 2001) and cell cycle control in tobacco (Espunya et al., 1999). We found two distinct alpha subunits and two distinct beta subunits in the purified protein complex(es) using a TAP-tagged CK2alpha subunit (Table 1, no. 20). This accounts for two of the three CK2alpha subunits and both of the CK2beta subunits in the rice genome, and the third CK2alpha subunit appears to contain a transit peptide indicating it is probably plastid localized. The two different alpha subunits must be in the same complex, but we cannot distinguish whether the different beta subunits occur in the same or different CK2 complexes. CK2beta subunits are regulated by protein degradation through a YRQALDMILD'cyclin destruction' consensus sequence (Allende and Allende, 1995). The rice CK2beta genes contain a related YDYALDLILD box at the analogous position in the CK2 protein, and our MS results found ubiquitin to be associated with the CK2 complex. This is consistent with the known ubiquitination of the 'cyclin destruction' consensus sequence in the cyclins and suggests at least some of the CK2beta is ubiquitinated in the CK2 complex.

\section{Mitogen activated protein kinases}

The yeast Ste20 kinase is a MAPKKKK that functions upstream of MAPK pathways involved both in mating (Dan 
et al., 2001) and osmotic stress responses (RamezaniRad, 2003). Rice contains over 20 MAPK-related protein kinases of unknown function that are distantly related to yeast Ste20 and presumably function in MAPK signaling pathways. The TAP-tagged rice Ste20-related protein kinase (Table 1, no. 12) was associated with a small amount, judged by peptide coverage, of both a MAPKK and an annexin protein. As discussed above, annexins are $\mathrm{Ca}^{2+}$-dependent proteins that bind to membrane phospholipids and are often involved in calcium signaling processes. Taken together, these findings suggest that these three proteins might be involved in a membraneassociated, $\mathrm{Ca}^{2+}$-dependent MAPK signaling cascade.

\section{Wall-associated kinases}

The wall-associated kinases (WAK) are transmembrane RLKs that are involved in plant defense, heavy metal responses and plant development (Verica and He, 2002), and show a calcium-dependent binding to polygalacturonic acid, oligogalacturonides and pectins (Decreux and Messiaen, 2005). The chloroplast-localized oxygenevolving protein 2 has been shown to be phosphorylated by the cytoplasmic kinase domain of Arabidopsis WAK1 (Yang et al., 2003). We found a rice WAK1-related protein interaction with rice oxygen evolving enhancer protein 3 , as well as an aspartic protease, a hydroxyproline-rich glycoprotein and additional proteins (Table 1, no. 2). Rice WAK2 interacted with a calmodulin-binding motif protein, a putative disease resistance protein, and a phospholipase $C$ protein (Table 1, no. 1), indicating interactions with cell signaling components. A third WAK (Table 1, no. 23), also closely related to Arabidopsis WAK2, showed binding to alpha and beta tubulin actin, an armadillo/beta-catenin-like repeat protein, and a protein kinase of unknown function, suggesting an interaction with cytoskeletal components (Coates, 2003). An additional rice WAK2-related protein (Table 1, no. 24) was purified without recovering any interacting proteins.

\section{Conclusions}

Additional interacting proteins are present in Table 1 that lack supporting data from other organisms. In cases where no orthologous interactions are known, the question arises on how to further prove that the identified interactions are valid. The usual validation for protein interactions is a co-immunoprecipitation of the proteins, with antibodies to the proteins or their epitope-tagged versions. However, other than using different affinity beads, this method is essentially similar to the first step of the TAP tag procedure and both suffer from the same potential problems: interaction of contaminating proteins with the beads or the tagged proteins in vitro after the cells are disrupted. This would suggest that the next step would be to demonstrate that the interaction occurs in vivo. This could be done by yeast two-hybrid interaction methods or in vivo imaging methods in plants such as fluorescence resonance energy transfer (Piehler, 2005). Alternatively, the TAP method could be modified to include a denaturing purification step after protein crosslinking in vivo (Rohila et al., 2004) to require covalent association of co-purifying proteins. This might be feasible as we have found that the order of the TAP purification affinity steps can be successfully reversed (JSR, unpublished data). The first calmodulin binding/release step could be followed by a denaturing IgG step as the protein A-lgG interaction is stable in the presence of low amounts of SDS, although we have not tested this protocol as yet.

Our results demonstrate the utility of the TAP method for discovering protein-protein interactions in cereals. The identification of a protein in a complex provides more detailed information about its function than a homology-based comparison, particularly when the role of the orthologous complex is known in other species. In several instances, we found multiple isoforms of a protein family in the isolated complexes. This data should help identify which gene family members appear to have similar or redundant functions. The close evolutionary relationship of rice with the other cereals (Rensink and Buell, 2004) allows for extension of these results to those crops.

The two most critical variables for the successful isolation of protein complexes are the abundance and stability of the complex. These variables are influenced by the experimental choices of whether to overexpress the protein, reduce the competition from endogenous proteins, test both $\mathrm{N}$ - and $\mathrm{C}$-terminal fusions of the protein, use different affinity tags, and to choose a tissue source with sufficient expression and amounts of tissue. Further improvements in these methods to increase the amount of recoverable protein complexes should improve the success rate of identifying interacting proteins.

\section{Experimental procedures}

Reverse transcription-PCR, cDNA cloning and rice transformation

Total RNA was isolated from Nipponbare rice leaves using Trizol reagent (Invitrogen). First strand cDNA was synthesized using RETROscript ${ }^{\circledR}$ reverse transcriptase (Ambion, Austin, TX, USA). PCR was performed with Taq polymerase to quantify the amount of cDNA. cDNA products to be cloned were amplified using nested primers and Vent DNA polymerase (New England Biolabs, Ipswich, MA, USA). The internal set of PCR primers contained attB1 and attB2 sequences to allow for BP cloning (Gateway ${ }^{\circledR}$ of the PCR product into pDONR207. These internal primers contained homology to the ATG start codon and the termination codon to create a cDNA clone without a 5 ' untranslated region and less than $20 \mathrm{bp}$ of $3^{\prime}$ untranslated region. The 5 ' open reading frame was consistent with the upstream Gateway ${ }^{\circledR}$ reading frame (AttB1 forward: 
5'-ggggacaagtttgtacaaaaaagcaggctcaacc ATG, where the ATG is the translation initiation codon). In the case of the RLK cDNAs, the extracellular and transmembrane domains were not included and the primer homolog was to the beginning of the cytoplasmic domain, and an ATG translation initiation codon was added. The sequences of the cDNAs were determined to be consistent with the release 3 gene models of The Institute of Genomic Research (http://www.tigr.org/tdb/e2k1/osa1/) and will be deposited in GenBank. The cDNA clones in the BP vector were recombined into the final Ubi-NTAP-1300 vector using an LR reaction $\left(\right.$ Gateway ${ }^{\circledR}$ ). Ubi-NTAP-1300 is a pCAMBIA-1300 (AF234296) derivative with an additional expression cassette containing the maize ubiquitin promoter and a nopaline synthase 3' polyadenylation region (Nos 3'; Chern et al., 2005), to which the NTAP/Gateway ${ }^{\circledR}$ cassette was added (Rohila et al., 2004). Rice transformation of seed-derived calli was carried out as described (Chern et al., 2005).

\section{Tandem affinity purification}

Shoots of 6- to 8-week-old T1 transgenic rice plants were harvested for protein purifications essentially as described by Rohila et al. (2004). In short, 50-60 g (fresh weight) of rice shoots was ground to a fine powder in liquid nitrogen using a high-speed blender (Waring Laboratory, Torrington, $\mathrm{CT}, \mathrm{USA}$ ). Crude protein extracts were prepared in three-four volumes of protein extraction buffer [20 mm Tris-HCl, pH 8.0, $150 \mathrm{~mm}$ $\mathrm{NaCl}, 0.1 \%$ IGEPAL (Sigma, Saint Louis, MO, USA), $2.5 \mathrm{~mm}$ EDTA, $2 \mathrm{~mm}$ benzamidine, $10 \mathrm{~mm} \beta$-mercaptoethanol, $20 \mathrm{~mm} \mathrm{NaF}, 1 \mathrm{~mm}$ phenylmethanesulfonylfluoride (PMSF), 1\% Protease cocktail (Sigma), $10 \mu \mathrm{m}$ leupeptin (Sigma), and $10 \mu \mathrm{m}$ 3,4-dichloroisocoumarin (Sigma)]. The extract was passed through a fine sieve, filtered through two layers of miracloth, and centrifuged twice at a speed of 30,000 $\mathrm{g}$ for $20 \mathrm{~min}$ at $4^{\circ} \mathrm{C}$. The cleared supernatant was mixed with $100-150 \mu \mathrm{l}$ of IgG Sepharose beads (Amersham Biosciences, Piscataway, NJ, USA) and incubated at $4^{\circ} \mathrm{C}$ for $2 \mathrm{~h}$ on a Labquake ${ }^{\circledR}$ Rotisserie (Barnstead Thermolyne Corporation, Dubuque, IA, USA). After centrifugation at $200 \mathrm{~g}$ for 2$3 \mathrm{~min}$, IgG supernatant was discarded and the collected IgG beads were washed in $30 \mathrm{ml}$ extraction buffer lacking protease inhibitors. The washed beads were loaded onto a disposable polyprep chromatography column (Bio-Rad Laboratories, Hercules, CA, USA) and washed with $10 \mathrm{ml}$ TEV cleavage buffer ( $10 \mathrm{~mm}$ Tris- $\mathrm{HCl} \mathrm{pH} \mathrm{8.0,150} \mathrm{mm} \mathrm{NaCl}$, $0.1 \%$ IGEPAL, $0.5 \mathrm{~mm}$ EDTA, $1 \mathrm{~mm}$ DTT). The TAP-tagged proteins were released by digestion with $150 \mathrm{U}$ of rTEV protease (Invitrogen) in TEV cleavage buffer containing $1 \mu \mathrm{m} \mathrm{E}-64$ protease inhibitor for $1 \mathrm{~h}$ at $16^{\circ} \mathrm{C}$. The TEV cleaved eluate was adjusted to $2 \mathrm{~mm} \mathrm{CaCl}_{2}$ and diluted in 3 volumes of calmodulin-binding buffer (CBB: $10 \mathrm{~mm} \beta$-mercaptoethanol; $10 \mathrm{~mm}$ Tris- $\mathrm{HCl} \mathrm{pH} 8.0 ; 150 \mathrm{~mm} \mathrm{NaCl} ; 1 \mathrm{~mm}$ Mg-acetate; $1 \mathrm{~mm}$ imidazole; $2 \mathrm{~mm} \mathrm{CaCl}$; $0.1 \%$ IGEPAL) and incubated with $100 \mu \mathrm{l}$ of calmodulin-agarose beads (Stratagene, La Jolla, CA, USA) for $1 \mathrm{~h}$ at $4^{\circ} \mathrm{C}$. The calmodulin-agarose beads were washed in $30 \mathrm{ml}$ of calmodulin binding buffer and the proteins were eluted with buffer containing $10 \mathrm{~mm} \beta$-mercaptoethanol, $10 \mathrm{~mm}$ Tris- $\mathrm{HCl} \mathrm{pH} 8.0,150 \mathrm{~mm} \mathrm{NaCl}, 1 \mathrm{~mm}$ Mg-acetate, $1 \mathrm{~mm}$ imidazole, $2 \mathrm{~mm}$ EGTA, 0.1\% IGEPAL. The proteins in the eluate were precipitated with trichloroacetic acid and loaded onto a $415 \%$ gradient polyacrylamide gel for SDS-PAGE. Proteins were visualized by fluorescence staining (Sypro Ruby; Bio-Rad Laboratories).

\section{Calmodulin-bead assay and Western blots}

Bead assays for TAP-tagged protein expression analyses were performed as described previously (Rohila et al., 2004) except that the protein extracts were initially prepared in CBB lacking $\mathrm{Mg}^{2+}$ and $\mathrm{Ca}^{2+}$. One millimolar Mg-acetate and $2 \mathrm{~mm} \mathrm{CaCl}_{2}$ were then added to the cleared extracts.
The proteins bound to the calmodulin-agarose beads were also used for Western blot analysis. The bound proteins were released by boiling in SDS loading buffer for $5 \mathrm{~min}$ and the supernatant collected after centrifugation at $20,000 \mathrm{~g}$ for $2 \mathrm{~min}$. The proteins in the supernatant were separated on a $10 \%$ SDS-polyacrylamide gel, transferred onto a PVDF membrane (Pierce, Rockford, IL, USA) and the protein A domains of the TAP tag were detected with an antibody:peroxidase conjugate (PAP, Sigma) as described by (Rivas et al., 2002).

\section{Mass spectrometry}

The Sypro Ruby stained bands from the SDS-PAGE gel were excised and the digested peptides subjected to LC/MS as described (Kayser et al., 2004; Rohila et al., 2004). Briefly, gel pieces were digested by trypsin (no. V5111, Promega, Madison, WI, USA) and digested peptides were extracted in $5 \%$ formic acid $/ 50 \%$ acetonitrile and separated using a C18 reversed phase LC column (Dionex, Sunnyvale, CA, USA). A Q-TOF Ultima tandem mass spectrometer (Waters, Milford, MA, USA) with electrospray ionization was used to analyze the eluting peptides. The system was user controlled employing masslynx software (v4.0, Waters) in data-dependant acquisition mode with the following parameters: 1-sec survey scan (380-1,900 Da) followed by up to three 2.4-sec MS/MS acquisitions (60-1900 Da). The instrument was operated at a mass resolution of 8,000 . The instrument was calibrated using the fragment ion masses of doubly protonated Glu-fibrinopeptide.

The peak lists of MS/MS data were generated using Distiller (Matrix Science, v1.9.0, London, UK) using charge state recognition and de-isotoping with the other default parameters for Q-TOF data. Database searches of the acquired MS/MS spectra were performed using Mascot (Matrix Science, v1.9.0, London, UK). Two databases were utilized. When using the MSDB database (a comprehensive, non-identical protein sequence database maintained by the Proteomics Department at the Hammersmith Campus of Imperial College London, which combines entries from TREMPL, SWISSPOT and GenBank), the taxonomy filter was set as Vindiplantae (green plants). The rice genome sequences (http://www.tigr.org/tbe/e2k1/osa1/) were also utilized. Search parameters used were: no restrictions on protein molecular weight or pl, enzymatic specificity was set to trypsin, and methionine oxidation and phosphorylation were allowed as variable peptide modifications. Mass accuracy settings were $0.15 \mathrm{Da}$ for peptide mass and 0.12 Da for fragment ion masses. mascot peptide scores equal to or higher than the 'identity'mascot score (typically 39-41) for that peptide were required for a peptide to be considered a valid identification of a protein. The interaction data and additional information about rice protein kinases are available on the project website (http://rkd.ucdavis.edu/).

All of the MS/MS spectra of peptides that were assigned by mascot as potentially having been phosphorylated were examined manually to verify the assignment. The concurrent loss of $\mathrm{H}_{3} \mathrm{PO}_{4}(98 \mathrm{Da})$ along with the expected peptide backbone fragmentation was used to validate the mascot assignment.

\section{Supplemental Materials are appended to this edition, fol- lowing the References.}

\section{Acknowledgements}

We thank Dr. Shoshi Kikuchi for early access to the rice fulllength cDNA database, and Dr. Ouyang Shu and Dr. Robin 
Buell for help with the rice gene annotation. We also thank Karin van Dijk, Alan Christensen, and Pedro Rocha for critical reading of the manuscript. The MS facility is supported in part by National Institutes of Health Grant P20 RR15635 from the Centres of Biomedical Research Excellence Program of the National Center for Research Resources, National Cancer Institute Cancer Center Support Grant P30 CA36727, and the Nebraska Research Initiative. This work was supported by the Nebraska Research Initiative and the National Science Foundation (DBI-0217312) to MEF.

\section{References}

Allende, J.E. and Allende, C.C. (1995) Protein kinases. 4. Protein kinase CK2: an enzyme with multiple substrates and a puzzling regulation. FASEB J. 9, 313-323.

An, S., Park, S., Jeong, D.H. et al. (2003) Generation and analysis of end sequence database for T-DNA tagging lines in rice. Plant Physiol. 133, 2040-2047.

Bachmann, M., Huber, J.L., Liao, P.C., Gage, D.A. and Huber, S.C. (1996) The inhibitor protein of phosphorylated nitrate reductase from spinach (Spinacia oleracea) leaves is a 143-3 protein. FEBS Lett. 387, 127-131.

Bibby, A.C. and Litchfield, D.W. (2005) The multiple personalities of the regulatory subunit of protein kinase CK2: CK2 dependent and CK2 independent roles reveal a secret identity for CK2beta. Int. J. Biol. Sci. 1, 67-79.

Burack, W.R. and Shaw, A.S. (2000) Signal transduction: hanging on a scaffold. Curr. Opin. Cell Biol. 12, 211-216.

Chen, M., Chory, J. and Fankhauser, C. (2004) Light signal transduction in higher plants. Annu. Rev. Genet. 38, 87117.

Chern, M., Fitzgerald, H.A., Canlas, P.E., Navarre, D.A. and Ronald, P.C. (2005) Overexpression of a rice NPR1 homolog leads to constitutive activation of defense response and hypersensitivity to light. Mol. Plant Microbe Interact. $18,511-520$

Chinnusamy, V., Schumaker, K. and Zhu, J.K. (2004) Molecular genetic perspectives on cross-talk and specificity in abiotic stress signalling in plants. J. Exp. Bot. 55, 225-236.

Christensen, A.H. and Quail, P.H. (1996) Ubiquitin promoterbased vectors for high-level expression of selectable and/ or screenable marker genes in monocotyledonous plants. Transgenic Res. 5, 213-218.

Coates, J.C. (2003) Armadillo repeat proteins: beyond the animal kingdom. Trends Cell Biol. 13, 463-471.

Dan, I., Watanabe, N.M. and Kusumi, A. (2001) The Ste20 group kinases as regulators of MAP kinase cascades. Trends Cell Biol. 11, 220-230.

Daniel, X., Sugano, S. and Tobin, E.M. (2004) CK2 phosphorylation of CCA1 is necessary for its circadian oscillator function in Arabidopsis. Proc. Natl Acad. Sci. USA, 101, 32923297.

Decreux, A. and Messiaen, J. (2005) Wall-associated kinase WAK1 interacts with cell wall pectins in a calcium-induced conformation. Plant Cell Physiol. 46, 268-278.
Diehl, J.A., Yang, W., Rimerman, R.A., Xiao, H. and Emili, A. (2003) Hsc70 regulates accumulation of cyclin D1 and cyclin D1-dependent protein kinase. Mol. Cell Biol. 23, 17641774.

Dievart, A. and Clark, S.E. (2003) Using mutant alleles to determine the structure and function of leucine-rich repeat receptor-like kinases. Curr. Opin. Plant Biol. 6, 507-516.

Espunya, M.C., Combettes, B., Dot, J., Chaubet-Gigot, N. and Martinez, M.C. (1999) Cell-cycle modulation of CK2 activity in tobacco BY-2 cells. Plant J. 19, 655-666.

Etheridge, N., Chen, Y.F. and Schaller, G.E. (2005) Dissecting the ethylene pathway of Arabidopsis. Brief Funct. Genomic. Proteomic. 3, 372-381.

Fabian-Marwedel, T., Umeda, M. and Sauter, M. (2002) The rice cyclin-dependent kinase-activating kinase $\mathrm{R} 2$ regulates S-phase progression. Plant Cell, 14, 197-210.

Fan, L.M., Zhao, Z. and Assmann, S.M. (2004) Guard cells: a dynamic signaling model. Curr. Opin. Plant Biol. 7, 537546.

Ferl, R.J. (2004) 14-3-3 proteins: regulation of signal-induced events. Physiol. Plant. 120, 173-178.

Forler, D., Kocher, T., Rode, M., Gentzel, M., Izaurralde, E. and Wilm, M. (2003) An efficient protein complex purification method for functional proteomics in higher eukaryotes. Nat. Biotechnol. 21, 89-92.

Gavin, A.C., Bosche, M., Krause, R. et al. (2002) Functional organization of the yeast proteome by systematic analysis of protein complexes. Nature, 415, 141-147.

Gerke, V., Creutz, C.E. and Moss, S.E. (2005) Annexins: linking $\mathrm{Ca} 2+$ signalling to membrane dynamics. Nat. Rev. Mol. Cell Biol. 6, 449-461.

Gingras, A.C., Aebersold, R. and Raught, B. (2005) Advances in protein complex analysis using mass spectrometry. J. Physiol. 563, 11-21.

Goff, S.A., Ricke, D., Lan, T.H. et al. (2002) A draft sequence of the rice genome (Oryza sativa L. ssp. japonica). Science, 296, 92-100.

Hiei, Y., Ohta, S., Komari, T. and Kumashiro, T. (1994) Efficient transformation of rice (Oryza sativa L.) mediated by Agrobacterium and sequence analysis of the boundaries of the T-DNA. Plant J. 6, 271-282.

Ho, Y., Gruhler, A., Heilbut, A. et al. (2002) Systematic identification of protein complexes in Saccharomyces cerevisiae by mass spectrometry. Nature, 415, 180-183.

Ichimura, T., Isobe, T., Okuyama, T., Yamauchi, T. and Fujisawa, H. (1987) Brain 14-3-3 protein is an activator protein that activates tryptophan 5-monooxygenase and tyrosine 3-monooxygenase in the presence of $\mathrm{Ca} 2+$,calmodulin-dependent protein kinase II. FEBS Lett. 219, 79-82.

Inze, D. (2005) Green light for the cell cycle. EMBO J. 24 657-662.

IRGSP (2005) The map-based sequence of the rice genome. Nature, 436, 793-800.

Kayser, J.P., Vallet, J.L. and Cerny, R.L. (2004) Defining parameters for homology-tolerant database searching. J. Biomol. Tech. 15, 285-295. 
Kikuchi, S., Satoh, K., Nagata, T. et al. (2003) Collection, mapping, and annotation of over 28,000 cDNA clones from japonica rice. Science, 301, 376-379.

Koroleva, O.A., Tomlinson, M., Parinyapong, P., Sakvarelidze, L., Leader, D., Shaw, P. and Doonan, J.H. (2004) CycD1, a putative G1 cyclin from Antirrhinum majus, accelerates the cell cycle in cultured tobacco BY-2 cells by enhancing both G1/S entry and progression through S and G2 phases. Plant Cell, 16, 2364-2379.

Larochelle, S., Chen, J., Knights, R., Pandur, J., Morcillo, P., Erdjument-Bromage, H., Tempst, P., Suter, B. and Fisher, R.P. (2001) T-loop phosphorylation stabilizes the CDK7-cyclin H-MAT1 complex in vivo and regulates its CTD kinase activity. EMBO J. 20, 3749-3759.

Lee, S., Lee, E.J., Yang, E.J., Lee, J.E., Park, A.R., Song, W.H. and Park, O.K. (2004) Proteomic identification of annexins, calcium-dependent membrane binding proteins that mediate osmotic stress and abscisic acid signal transduction in Arabidopsis. Plant Cell, 16, 1378-1391.

Lee, K.M., Miklos, I., Du, H. et al. (2005) Impairment of the TFIIH-associated CDK-activating kinase selectively affects cell cycle-regulated gene expression in fission yeast. Mol. Biol. Cell, 16, 2734-2745.

Miki, D. and Shimamoto, K. (2004) Simple RNAi vectors for stable and transient suppression of gene function in rice. Plant Cell Physiol. 45, 490-495.

Miyao, A., Tanaka, K., Murata, K., Sawaki, H., Takeda, S., Abe, K., Shinozuka, Y., Onosato, K. and Hirochika, H. (2003) Target site specificity of the Tos 17 retrotransposon shows a preference for insertion within genes and against insertion in retrotransposon-rich regions of the genome. Plant Cell, 15, 1771-1780.

Morgan, D.O. (1995) Principles of CDK regulation. Nature, 374, 131-134.

Morrison, D.K. and Davis, R.J. (2003) Regulation of MAP kinase signaling modules by scaffold proteins in mammals. Annu. Rev. Cell Dev. Biol. 19, 91-118.

Nurnberger, T., Brunner, F., Kemmerling, B. and Piater, L. (2004) Innate immunity in plants and animals: striking similarities and obvious differences. Immunol. Rev. 198, 249266.

Paul, A.L., Sehnke, P.C. and Ferl, R.J. (2005) Isoform-specific subcellular localization among 14-3-3 proteins in Arabidopsis seems to be driven by client interactions. Mol. Biol. Cell, 16, 1735-1743.

Piehler, J. (2005) New methodologies for measuring protein interactions in vivo and in vitro. Curr. Opin. Struct. Biol. 15, 4-14.

Pratt, W.B., Krishna, P. and Olsen, L.J. (2001) Hsp90-binding immunophilins in plants: the protein movers. Trends Plant Sci. 6, 54-58.

Ramezani-Rad, M. (2003) The role of adaptor protein Ste50dependent regulation of the MAPKKK Ste11 in multiple signalling pathways of yeast. Curr. Genet. 43, 161-170.

Rensink, W.A. and Buell, C.R. (2004) Arabidopsis to rice. Applying knowledge from a weed to enhance our understanding of a crop species. Plant Physiol. 135, 622-629.
Rivas, S., Romeis, T. and Jones, J.D. (2002) The Cf-9 disease resistance protein is present in an approximately 420-kilodalton heteromultimeric membrane-associated complex at one molecule per complex. Plant Cell, 14, 689-702.

Rohila, J.S., Chen, M., Cerny, R. and Fromm, M.E. (2004) Improved tandem affinity purification tag and methods for isolation of protein heterocomplexes from plants. Plant J. 38 , 172-181.

Rolland, F. and Sheen, J. (2005) Sugar sensing and signalling networks in plants. Biochem. Soc. Trans. 33, 269-271.

Rowland, O., Ludwig, A.A., Merrick, C.J. et al. (2005) Functional analysis of Avr9/Cf-9 rapidly elicited genes identifies a protein kinase, ACIK1, that is essential for full Cf-9-dependent disease resistance in tomato. Plant Cell, 17, 295310.

Rubio, V., Shen, Y., Saijo, Y., Liu, Y., Gusmaroli, G., DineshKumar, S.P. and Deng, X.W. (2005) An alternative tandem affinity purification strategy applied to Arabidopsis protein complex isolation. Plant J. 41, 767-778.

Sallaud, C., Meynard, D., van Boxtel, J. et al. (2003) Highly efficient production and characterization of T-DNA plants for rice (Oryza sativa L.) functional genomics. Theor. Appl. Genet. 106, 1396-1408.

Sallaud, C., Gay, C., Larmande, P. et al. (2004) High throughput T-DNA insertion mutagenesis in rice: a first step towards in silico reverse genetics. Plant J. 39, 450-464.

Shiu, S.H., Karlowski, W.M., Pan, R., Tzeng, Y.H., Mayer, K.F. and Li, W.H. (2004) Comparative analysis of the receptorlike kinase family in Arabidopsis and rice. Plant Cell, 16, 1220-1234.

Takagi, Y., Masuda, C.A., Chang, W.H., Komori, H., Wang, D., Hunter, T., Joazeiro, C.A. and Kornberg, R.D. (2005) Ubiquitin ligase activity of TFIIH and the transcriptional response to DNA damage. Mol. Cell, 18, 237-243.

Takahashi, Y., Shomura, A., Sasaki, T. and Yano, M. (2001) $\mathrm{Hd} 6$, a rice quantitative trait locus involved in photoperiod sensitivity, encodes the alpha subunit of protein kinase CK2. Proc. Natl Acad. Sci. USA, 98, 7922-7927.

Tchieu, J.H., Fana, F., Fink, J.L. et al. (2003) The plantsP and plants T functional genomics databases. Nucleic Acids Res. 31, 342-344.

Teige, M., Scheikl, E., Eulgem, T., Doczi, R., Ichimura, K., Shinozaki, K., Dangl, J.L. and Hirt, H. (2004) The MKK2 pathway mediates cold and salt stress signaling in Arabidopsis. Mol. Cell, 15, 141-152.

Verica, J.A. and He, Z.H. (2002) The cell wall-associated kinase (WAK) and WAK-like kinase gene family. Plant Physiol. 129, 455-459.

Verkest, A., Manes, C.L., Vercruysse, S., Maes, S., Van Der Schueren, E., Beeckman, T., Genschik, P., Kuiper, M., Inze, D. and De Veylder, L. (2005) The cyclin-dependent kinase inhibitor KRP2 controls the onset of the endoreduplication cycle during Arabidopsis leaf development through inhibition of mitotic CDKA;1 kinase complexes. Plant Cell, 17, 1723-1736.

Vondriska, T.M., Pass, J.M. and Ping, P. (2004) Scaffold proteins and assembly of multiprotein signaling complexes. J. 
Mol. Cell Cardiol. 37, 391-397.

Yaffe, M.B., Rittinger, K., Volinia, S., Caron, P.R., Aitken, A., Leffers, H., Gamblin, S.J., Smerdon, S.J. and Cantley, L.C. (1997) The structural basis for 14-3-3:phosphopeptide binding specificity. Cell, 91, 961-971.

Yamaguchi, M., Umeda, M. and Uchimiya, H. (1998) A rice homolog of Cdk7/MO15 phosphorylates both cyclin-dependent protein kinases and the carboxy-terminal domain of RNA polymerase II. Plant J. 16, 613-619.

Yamaguchi, M., Fabian, T., Sauter, M., Bhalerao, R.P., Schrader, J., Sandberg, G., Umeda, M. and Uchimiya, H. (2000) Activation of CDK-activating kinase is dependent on interaction with $\mathrm{H}$-type cyclins in plants. Plant J. 24, 11-20.

Yang, E.J., Oh, Y.A., Lee, E.S., Park, A.R., Cho, S.K., Yoo, Y.J. and Park, O.K. (2003) Oxygen-evolving enhancer protein 2 is phosphorylated by glycine-rich protein 3/wall-associated kinase 1 in Arabidopsis. Biochem. Biophys. Res. Commun. 305, 862-868.

Yazaki, J., Kojima, K., Suzuki, K., Kishimoto, N. and Kikuchi, S. (2004) The Rice PIPELINE: a unification tool for plant functional genomics. Nucleic Acids Res. 32, D383-D387.

Yu, J., Hu, S., Wang, J. et al. (2002) A draft sequence of the rice genome (Oryza sativa L. ssp. indica). Science, 296, 79-92.

Zhang, J.Z., Creelman, R.A. and Zhu, J.K. (2004) From laboratory to field. Using information from Arabidopsis to engineer salt, cold, and drought tolerance in crops. Plant Physiol. $135,615-621$.

Zurita, M. and Merino, C. (2003) The transcriptional complexity of the TFIIH complex. Trends Genet. 19, 578-584.

\section{Supplemental Materials}

Numbers refer to the numbers in Table I of the text and to the lane numbers of Figure 2 in the text.

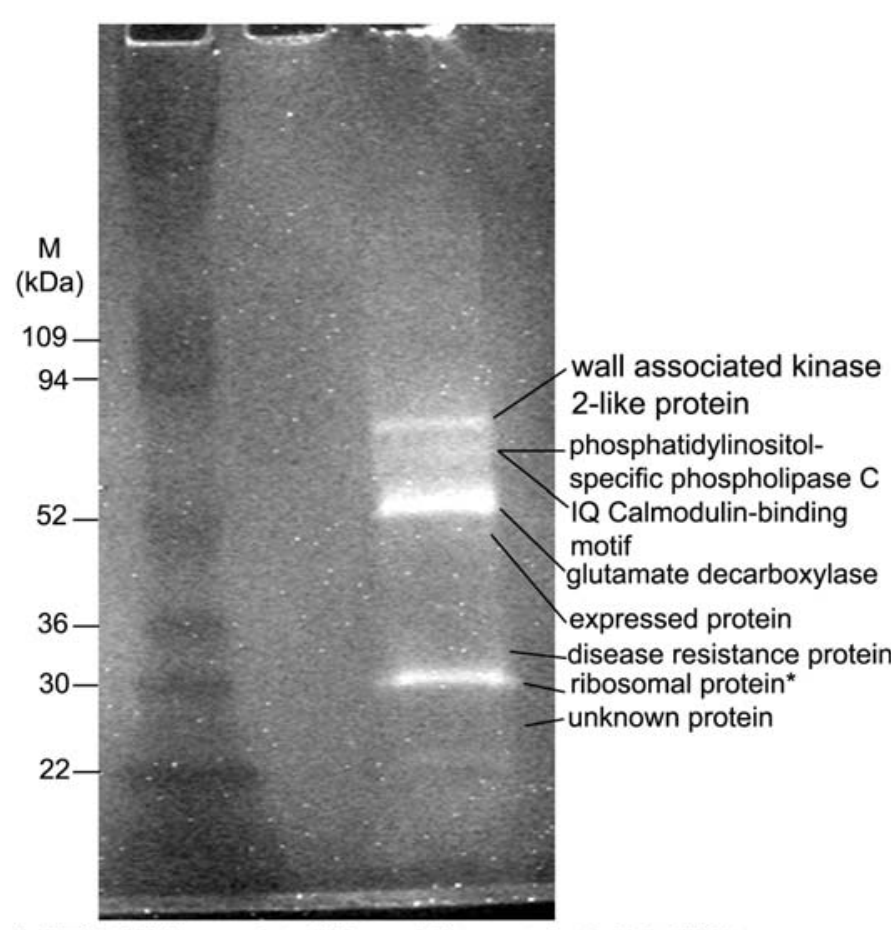

Figure S1. Fluorescently stained Sypro Ruby SDS-PAGE gels of tandem affinity purification (TAP)-purified wall associated kinase2-like protein (Os07g31130).

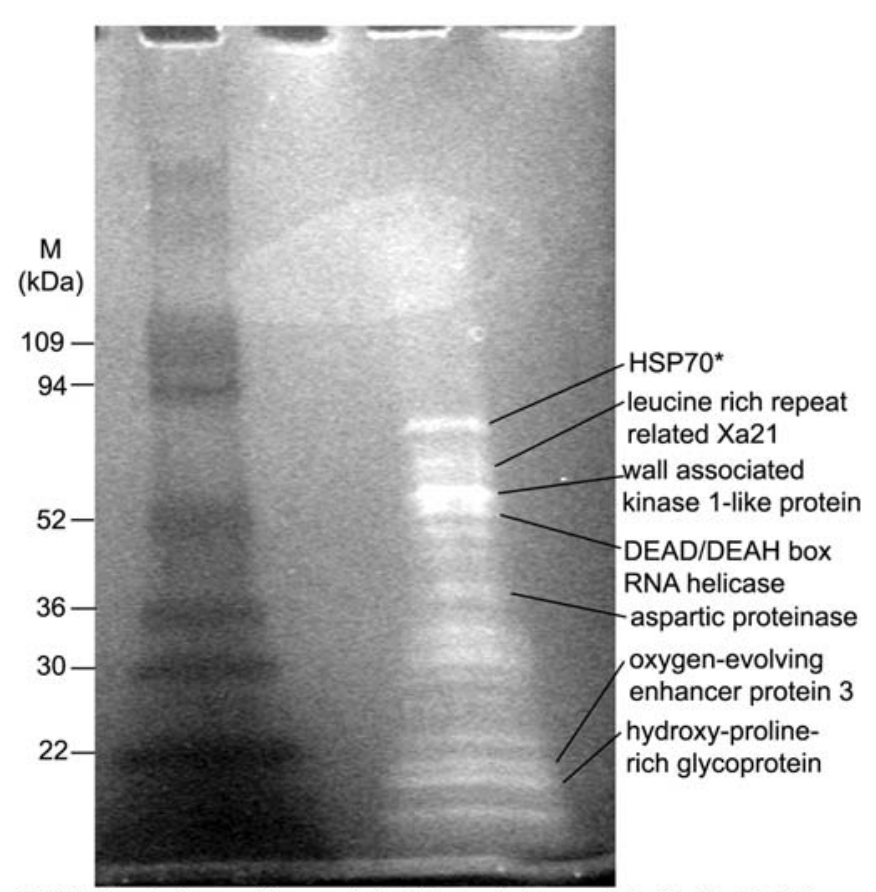

Figure S2. Fluorescently stained Sypro Ruby SDS-PAGE gels of tandem affinity purification (TAP)-purified putative wallassociated kinase1-like protein (Os10g10130). 


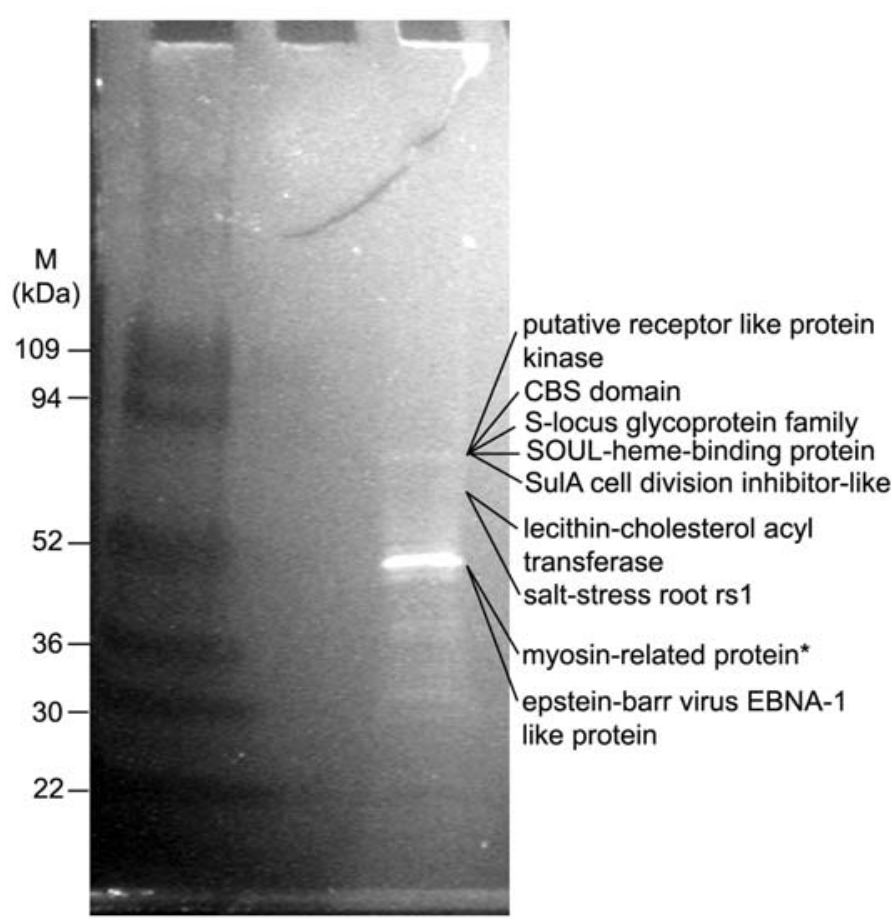

Figure S3. Fluorescently stained Sypro Ruby SDS-PAGE gels of tandem affinity purification (TAP)-purified putative receptorlike protein kinase (Os12g44090).

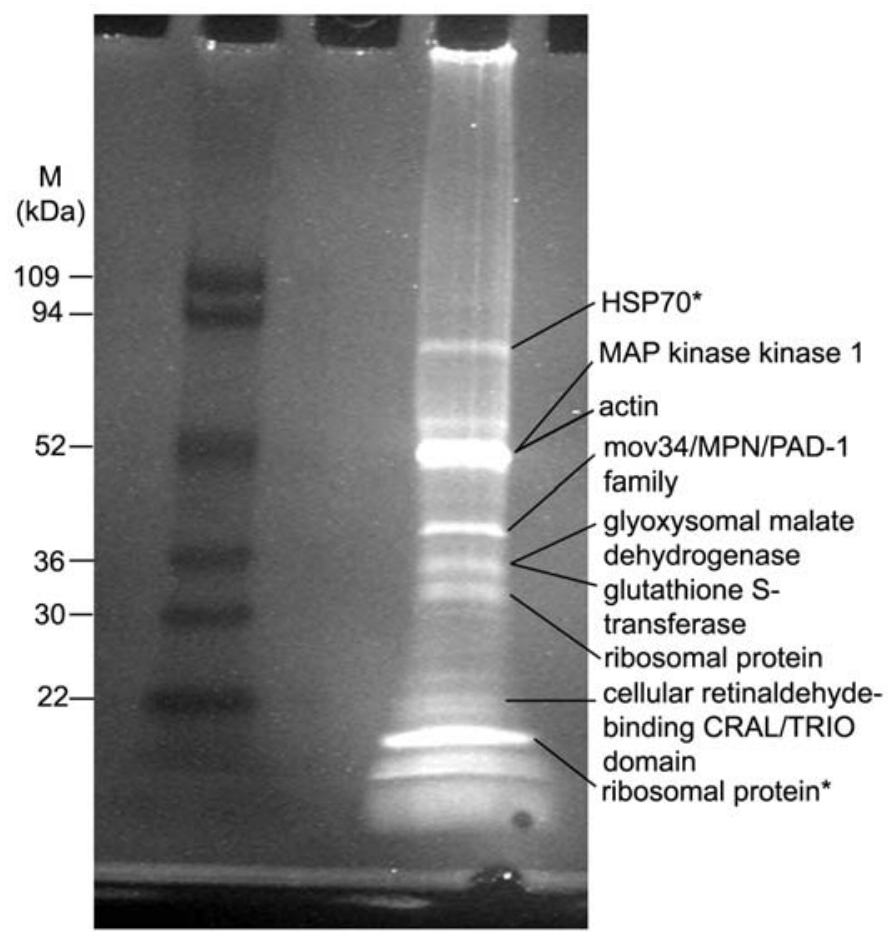

Figure S5. Fluorescently stained Sypro Ruby SDS-PAGE gels of tandem affinity purification (TAP)-purified MAP Kinase Kinase 1 (Os01g32660).

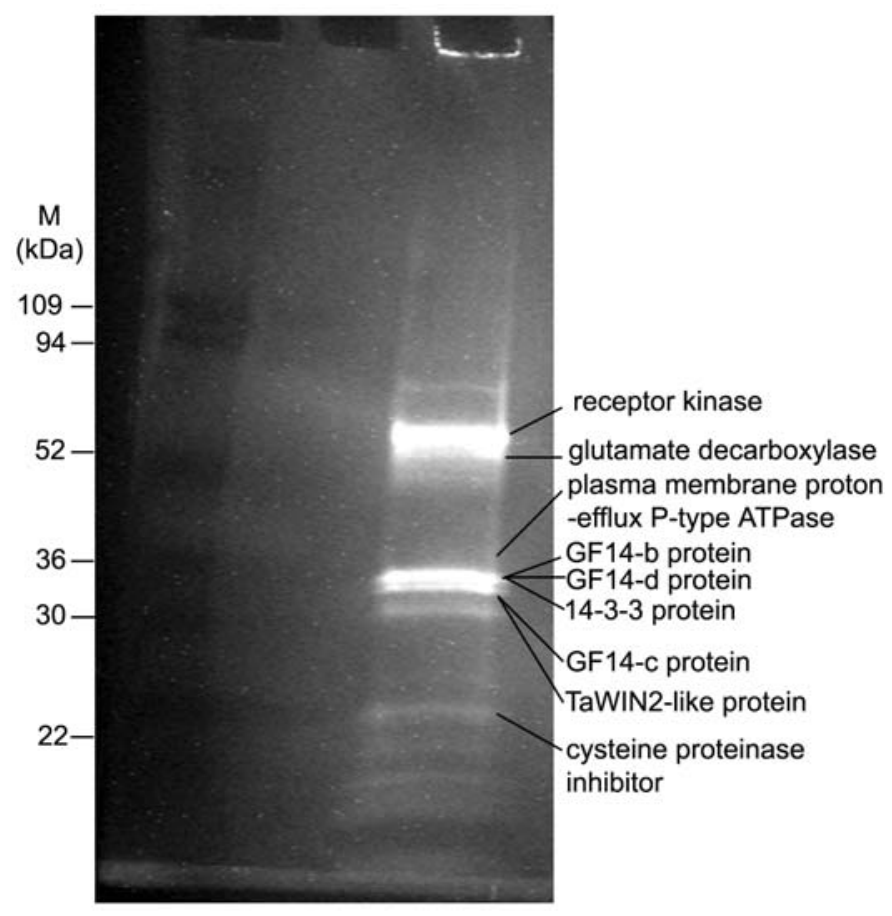

Figure S4. Fluorescently stained Sypro Ruby SDS-PAGE gels of tandem affinity purification (TAP)-purified receptor Kinase (Os03g61060).

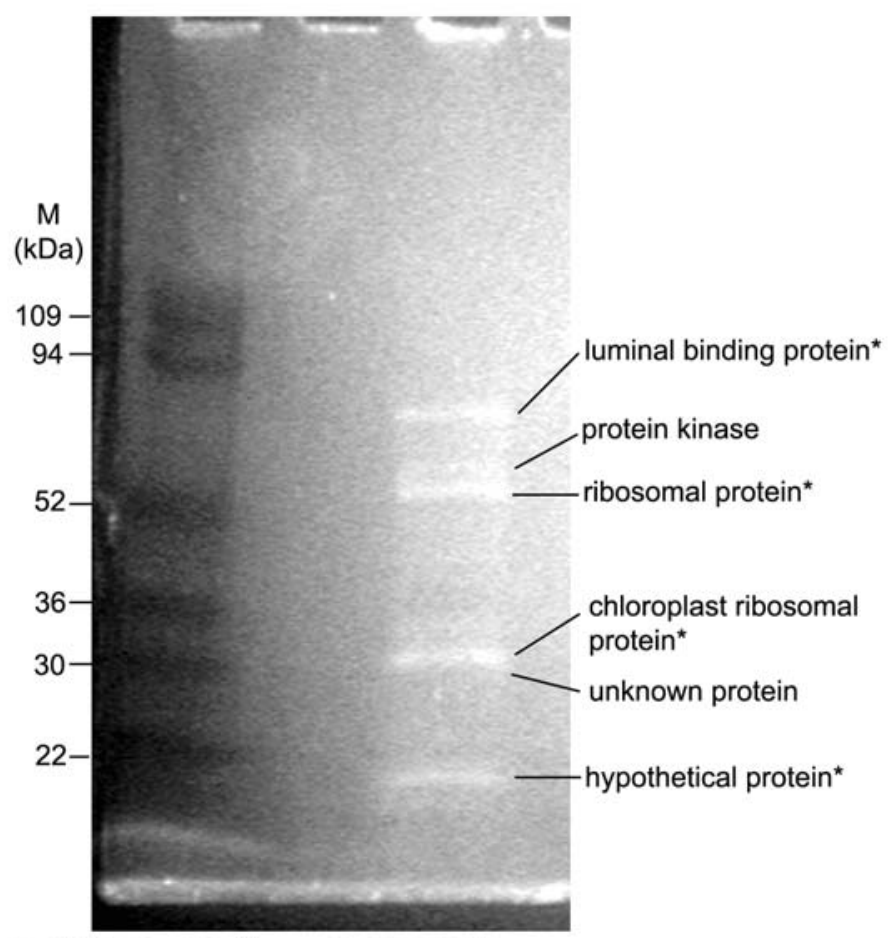

Figure S6. Fluorescently stained Sypro Ruby SDS-PAGE gels of tandem affinity purification (TAP)-purified protein kinase (Os03g05470). 
INTERACTIONS OF TAP-TAGgED PROTEIN KINASES IN RICE - Supplemental Materials

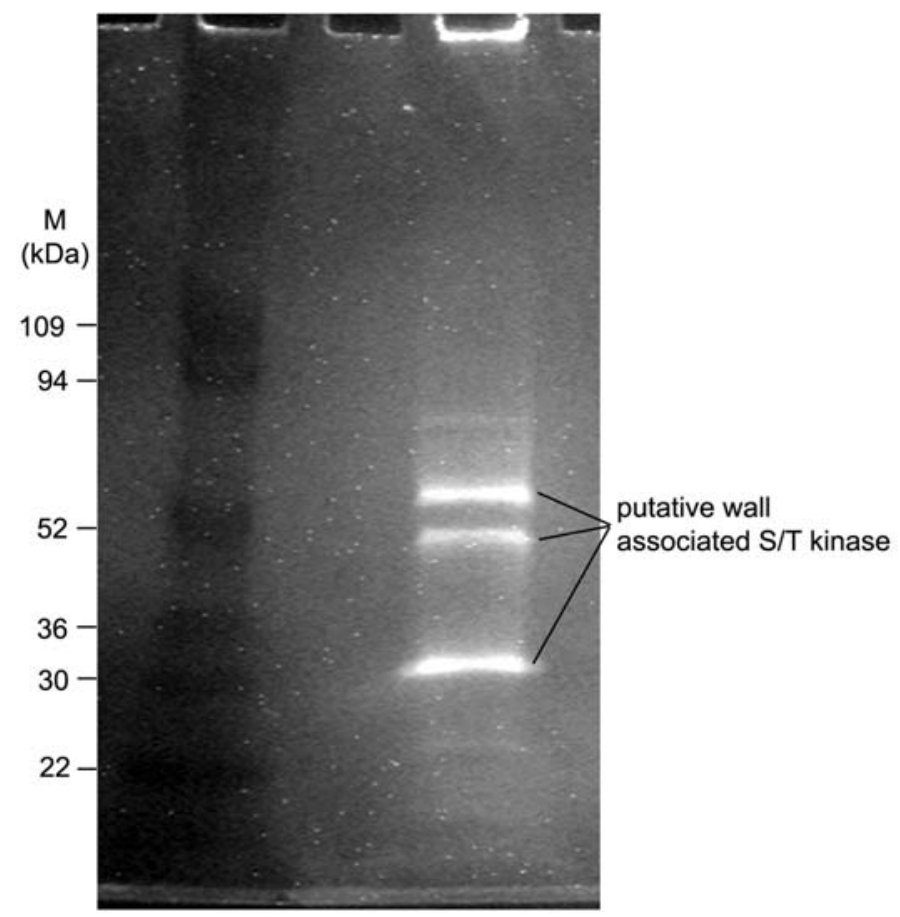

Figure S7. Fluorescently stained Sypro Ruby SDS-PAGE gels of tandem affinity purification (TAP)-purified putative wall-associated S/T kinase (Os09g29520). 
Table S1 Bead assay values or Western blot results for transgenic rice lines

1. Wall-associated kinase 2-like protein Os07g31130:

1797000

2. Putative wall-associated kinase 1-like protein Os10g10130:

5999000

3. Receptor like protein kinase Os12g44090:

5999000

4. Receptor kinase Os03g61060:

5999000

5. Receptor-like protein Kinase 4 Os07g35010:

860400

6. MAP Kinase Kinase 1 Os01g32660:

4999000

7. CDC2+/CDC28-related protein kinase Os05g32600:

*ND, western blot positive

8. Receptor like kinase Os01g02580:

1174700

9. Receptor S/T Kinase Os01g02310:

2185000

10. Receptor-like protein Kinase 4 Os07g43570:

2420000

11. Cyclin-dependent kinase B1-1 Os01g67160:

4999000

12. Ste20-related protein kinase Os10g37480:

1783000

13. rCDC2-1 Os03g02680:

4999000

14. SPK-3 Os01g64970:

4999000

15. Protein kinase Os01g10450:

4999000

16. Receptor S/T protein kinase Os01g01410:

2909000

17. Casein kinase Os01g38950:

350900

18. S-receptor kinase Os01g12410:

5553000

19. Protein Kinase Os08g12750:

8260

20. Casein Kinase II (CK2) alpha sub-unit Os07g02350:

*ND, western blot positive

21. Protein kinase APK1B Os01g41870:

5999000

22. Protein Kinase Os03g05470:

5999000

23. Wall associated protein kinase Os11g46950:

5999000

24. Putative wall-associated S/T Kinase Os09g29520: 5999000

25. Putative S/T Kinase Os07g48100:

3645000

26. CBL-interacting protein kinase 2 Os01g10890:

5999000

27. LRR receptor like protein kinase Os01g02040:

5999000

28. Wall-associated kinase 2-like protein Os07g31190:

5999000

29. Receptor S/T Kinase Os01g02290:

5600000

30. S/T Prot kinase Mak Male germ cell associated kinase Os06g02550: 4400

31. Putative S/T protein kinase Os07g32400:

17280

32. Putative S/T protein kinase Os01g60910:

37760

33. MAP Kinase Os03g17700:

34. Protein Kinase A FC1 Os01g62080:

4999000

35. Receptor protein kinase Os07g38070:

positive

36. Receptor like Kinase Os01g02390:

20360

37. Shaggy-related protein kinase gamma Os01g14860:

5999000

38. SAPK2 GI: 22296424 :

$* N D$, western blot positive
4999000

39. Protein kinase Os03g50330:

40. Calcium dependent Protein Kinase Os07g22710:

*ND, western blot positive

5999000

41. Protein Kinase Os04g52860:

5999000

**ND, western blot positive means that no bead assay was performed but a western blot of the extracts gave a positive signal. The bead assay values and the western blot signals are qualitative not quantitative.

Calmodulin bead assay protocol (Rohila, Chen et al. 2004)

Protein extracts, prepared as described for the TAP-tag purification method were adjusted to $2 \mathrm{mM} \mathrm{CaCl}_{2}$ and diluted three fold into calmodulinbinding buffer (CBB: $10 \mathrm{~mm}$ ß-mercaptoethanol; $10 \mathrm{mM}$ Tris-HCl pH8.0; $150 \mathrm{mM} \mathrm{NaCl} ; 1 \mathrm{mM} \mathrm{Mg-acetate;} 1 \mathrm{mM}$ imidazole; $2 \mathrm{mM} \mathrm{CaCl}$; $0.1 \%$ IGEPAL), bound to calmodulin-agarose beads (Stratagene) for $1 \mathrm{hr}$, and washed in CBB. The beads were then incubated in CBB supplemented with $30 \mathrm{mg} / \mathrm{ml}$ gelatin and a 1:5000 dilution of PAP conjugate (Sigma) for $1 \mathrm{hr}$ at room temperature. The beads were then washed three times with CBB and once with the substrate prior to incubation with the substrate (SUPERSIGNAL ${ }^{R}$ WEST PICO Chemiluminescent Substrate; Pierce). The signal was detected in a luminometer (LUMISTAR; BMG Labtechnologies).

\section{References}

Rohila, J. S., M. Chen, et al. (2004). „Improved tandem affinity purification tag and methods for isolation of protein heterocomplexes from plants.“ Plant J 38(1): 172-81. 